\title{
Water in Biomolecular Fluorescence Spectroscopy and Imaging: Side Effects and Remedies
}

\author{
Alexandre Fürstenberg*
}

\begin{abstract}
Historically, many of the classical organic fluorescent dyes were developed as laser dyes and characterized and optimized in organic solvents. Since then, fluorescence has, however, found a vast range of applications in the life sciences in which the fluorophores are usually surrounded by water and not by organic solvents. The omnipresence of water in biomolecular fluorescence spectroscopy and imaging leads to some unwanted but nonetheless unavoidable consequences on the photophysical properties of the dyes, which may impact the quality and complicate quantitative interpretation of the experiments. This paper discusses and illustrates with examples two such water-induced phenomena, namely chromophore aggregation in water and fluorescence quenching by water, as well as some ways to overcome them.
\end{abstract}

Keywords: Aggregation · Fluorescence · Heavy water · Quenching · Water

\section{Introduction}

Owing to their ability to detect molecular interactions, to resolve cellular and subcellular structures, to analyze their molecular composition, or to follow their dynamics in living systems, fluorescencebased methods have become indispensable tools for research in the life sciences. ${ }^{[1]}$ The success of fluorescence spectroscopy and imaging can be attributed to its very high sensitivity, to the diversity of parameters which can be followed (wavelength, intensity, lifetime, anisotropy), to the breadth of the possibilities of experimental implementation, and last but not least to the fact that it can be used directly in the native aqueous environment of the cell. Advanced fluorescence spectroscopy and imaging techniques nowadays allow experiments to be performed almost routinely at the level of individual molecules with a near-molecular spatial resolution, giving unprecedented information on cellular components, on local heterogeneities, on the dynamics of proteins, or on molecular interactions and localizations and their changes over time. ${ }^{[2]}$

Because the vast majority of cellular components and biomolecules do not display strong intrinsic fluorescence in the vis-

${ }^{*}$ Correspondence: Dr. A. Fürstenberg Institute for Physical and Theoretical Chemistry Goethe-University Frankfurt

Max-von-Laue-Str. 7

60438 Frankfurt am Main, Germany

and:

Laboratory of Chemical Biology and Signal Transduction, The Rockefeller University, New York, USA

E-mail: alexandre.furstenberg@gmail.com ible region of the spectrum, the prerequisite for most fluorescence experiments is that the molecules or structures of interest have to be labelled with an extrinsic fluorophore. In many applications, this external label is a small organic fluorophore belonging to one of the typical fluorescent dye classes such as rhodamines, cyanines, or oxazines, ${ }^{[3]}$ although fluorescent proteins which can be genetically incorporated into the target represent a popular alternative. ${ }^{[4]}$ There is nowadays a broad diversity of ways of coupling a fluorophore of choice to a molecule of interest be it genetically, by affinity labelling, or by direct chemical coupling thanks to the development over the past two decades of water-compatible bioorthogonal chemistries which are, in their most robust forms, quite readily usable by non-specialists. ${ }^{[1 b, 5]}$ The role of the fluorophore will usually be to highlight the location or the presence of the molecule of interest, but since fluorescence is sensitive to its environment, the fluorophore can also act as a sensor of its direct surroundings and report on local changes in polarity, $\mathrm{pH}$, interactions, and on the dynamics of the labelled biomolecule itself.[6]

One of the specificities of molecular spectroscopy and imaging applied to the life sciences is that the processes to be followed mostly take place either directly in an aqueous condensed phase or in an environment like a lipidic membrane which is in close proximity or direct contact with water molecules. Simply put, the fluorophore of interest will almost always be in direct contact with water or, if this is not the case, high water concentrations will usually not be far away. This simple fact will need to be taken into account when considering the fluorescence signal arising from the dye: since the photophysical properties of a fluorophore depend on its direct environment, it implies that the fluorescence intensity and lifetime will be often dominated by the presence of water as the solvent, or at least influenced by its proximity.

The omnipresence of water in biomolecular spectroscopy on one hand simplifies the picture as it seems to set a common ground to the majority of biomolecular fluorescence experiments (except, of course, those intended to investigate non-aqueous parts of the cell such as membranes). On the other hand, it also leads to some less expected but nonetheless unavoidable consequences which may impact the quality and complicate quantitative interpretation of the experiments. Chromophore aggregation in water and fluorophore quenching by water represent two such water-caused phenomena. Their nature, the problems they raise and some ways to overcome them are described and illustrated with some examples below.

\section{Dye Aggregation in Aqueous Solution}

Organic chromophoric structures tend to have rigid, planar moieties with conjugated double bonds at their core. They are therefore often not well soluble in water by themselves and require molecular groups and heteroatoms such as oxygen and nitrogen which favour hydrogen bonding to be added, or other functional groups which ionize in water such as sulfonates 
and carboxylic acids to improve their solubility in aqueous solution. Nonetheless, the planar chromophoric core is often less efficiently solvated by water than the regions of the dye which are designed to improve the interactions with water. In some cases, they will therefore reach better solvation by seeking interactions with a similar part of another dye molecule. As a consequence, dyes aggregate. An important part of the development of new and improved dyes for the life sciences during the past two decades has actually been to find ways to render existing dye core structures more water soluble in order to minimize aggregation while preserving or enhancing their intrinsic fluorescence properties and photostability. [7]

Aggregation can range from simple dimerization to higher order oligomerization and is mostly driven by a strong hydrophobic effect. A solution containing low-order aggregates such as dimers still appears as perfectly clear to the human eye in the sense that no scattering due to unsolubilized dye particles can be observed. Nonetheless aggregation can be detected spectroscopically as it strongly affects the photophysical properties of the dyes compared to the monomeric species, ${ }^{[8]}$ in particular the absorption and fluorescence band shape and position. Two geometries are often encountered and discussed in dye aggregates (Fig. 1): the dye molecules may aggregate parallel to each other in a sandwich-type, plane-toplane complex (so-called H-aggregates) or in a parallel, head-to-tail arrangement (socalled J-aggregates). The photophysical properties of such aggregates can be explained in terms of molecular exciton coupling theory. ${ }^{[9]}$ In a dimeric aggregate, the excitonic coupling between the two dye molecules leads to a splitting of the excited state into two levels. In $\mathrm{H}$-aggregates, the electronic transition to the upper excitonic state is allowed, but not that to the lower state. Therefore the absorption spectrum of the aggregate is shifted to the blue (higher energies) and the aggregate is non-fluorescent or very poorly fluorescent because of the fast internal conversion from the upper to the lower excitonic state, which is dark (forbidden transition from or to the ground state). In J-aggregates, the situation is reversed. The transition to the upper excitonic state is forbidden, whereas it is allowed to the lower excitonic state, leading to a red-shifted absorption spectrum and strong fluorescence emission from the aggregate.

Many dye classes have been shown to aggregate in water, including cyanines and rhodamines. ${ }^{[10]}$ Aggregation efficiency is usually concentration-dependent (the aggregation process is characterized by one or several thermodynamic equilibrium

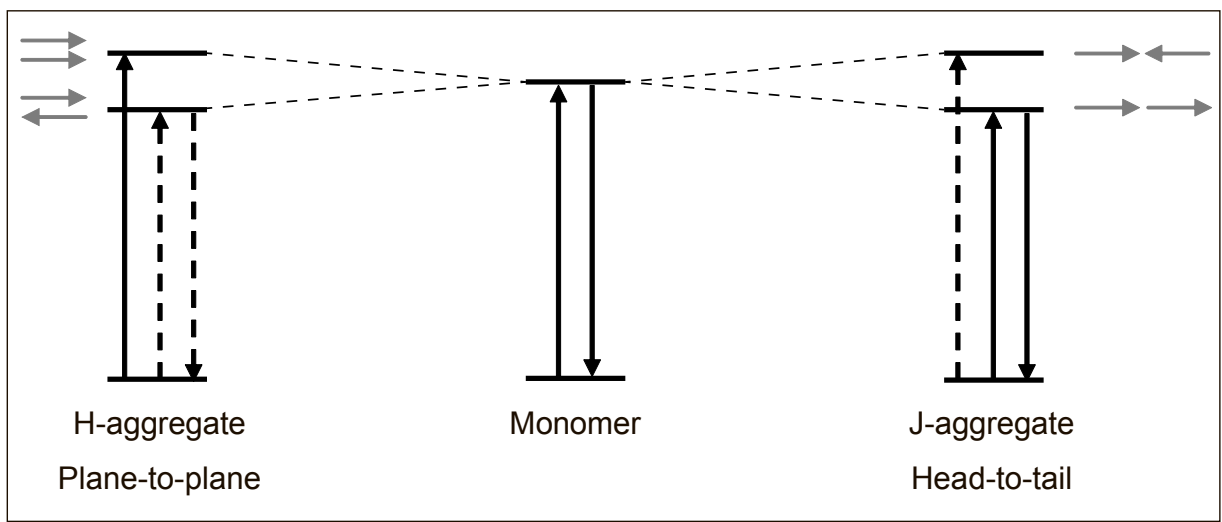

Fig. 1. Schematic representation of the result of excitonic coupling on the energy levels of aggregated and non-aggregated dye molecules depending on the relative spatial orientation of their transition dipole moments (small grey arrows). Plain vertical arrows represent allowed transitions and dashed arrows forbidden transitions (the transition dipole moments cancel out).

binding constants) and can occur at concentrations relevant to spectroscopic measurements, that is already in the low micromolar range, as illustrated with the dye YOSAC1. YOSAC1 belongs to a class of cyanine DNA intercalators (Fig. 2) whose discovery revolutionized molecular biology. ${ }^{[11]}$ The family of oxazole yellow (YO) and thiazole orange (TO) derivatives as well as their homodimeric relatives YOYO and TOTO display indeed very high extinction coefficients, show extremely low fluorescence when they are free in solution but form highly fluorescent and stable complexes with double-stranded DNA. ${ }^{[12]}$ These properties enabled the detection of DNA at a sensitivity only achieved until then with radioactive probes but without the danger inherent in the use of radioactivity. The principle explanation for the very high contrast between the free and the DNA-bound form of the dyes resides in an ultrafast, picosecond decay of the excited state of the free form through a large-amplitude torsional motion around the monomethine bridge connecting the benzoxazole and quinoline moieties of oxazole yellow. ${ }^{[13]}$ This isomerization process is sterically blocked upon intercalation of the dyes into DNA and, as a consequence, their fluorescence strongly increases. Disruption of dye aggregates upon intercalation into DNA however, also contributes to the fluorescence enhancement of the dyes. [14]

Fig. 3A displays the intensity-normalized absorption and fluorescence spectra of YOSAC1, a cyanine dye with a single positive charge, measured in different environments including aqueous buffer solution (PBS, aqueous phosphate buffer solution containing $137 \mathrm{mM} \mathrm{NaCl}$ ) and methanol.[14b,15] The absorption spectra are characterized by a maximum around $480 \mathrm{~nm}$ and a shoulder around $455 \mathrm{~nm}$ ascribed to a vibronic transition. ${ }^{[13]}$ In water however, the height of the shoulder increases with rising YOSAC1 concentration in the $1-50 \mu \mathrm{M}$ range (Fig. 3B, top), indicating that the dye undergoes intermolecular H-aggregation. ${ }^{[15]}$ This phenomenon is even more striking at higher ionic strength (PBS) and $200 \mu \mathrm{M}$ dye concentration. Aggregation is further confirmed by the shape of the fluorescence spectrum of YOSAC1 in aqueous solution (Fig. 3A): whereas the emission spectrum in organic solvents is the mirror image of the absorption spectrum, it is broad and red-shifted in PBS.

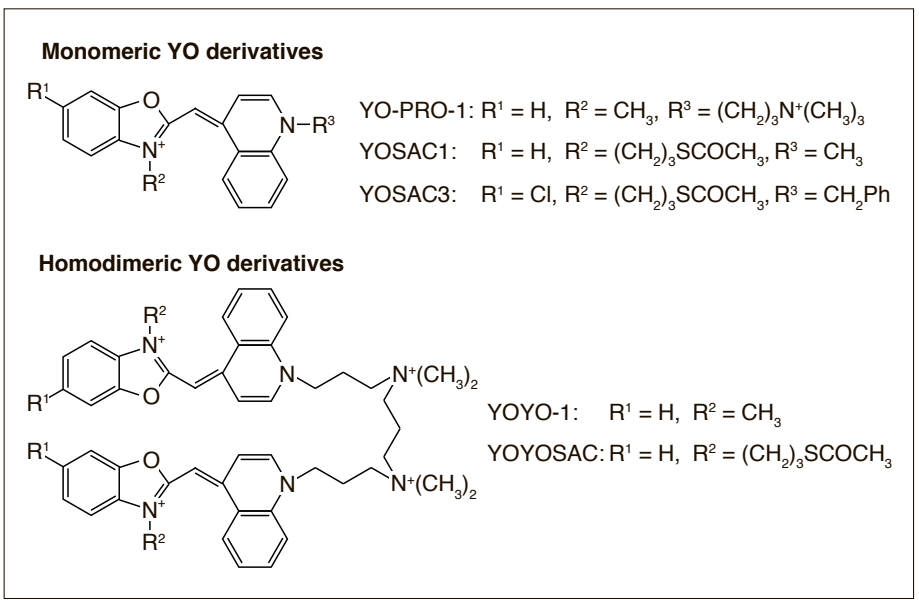

Fig. 2. Molecular structure of some oxazole yellow (YO) derivatives. 

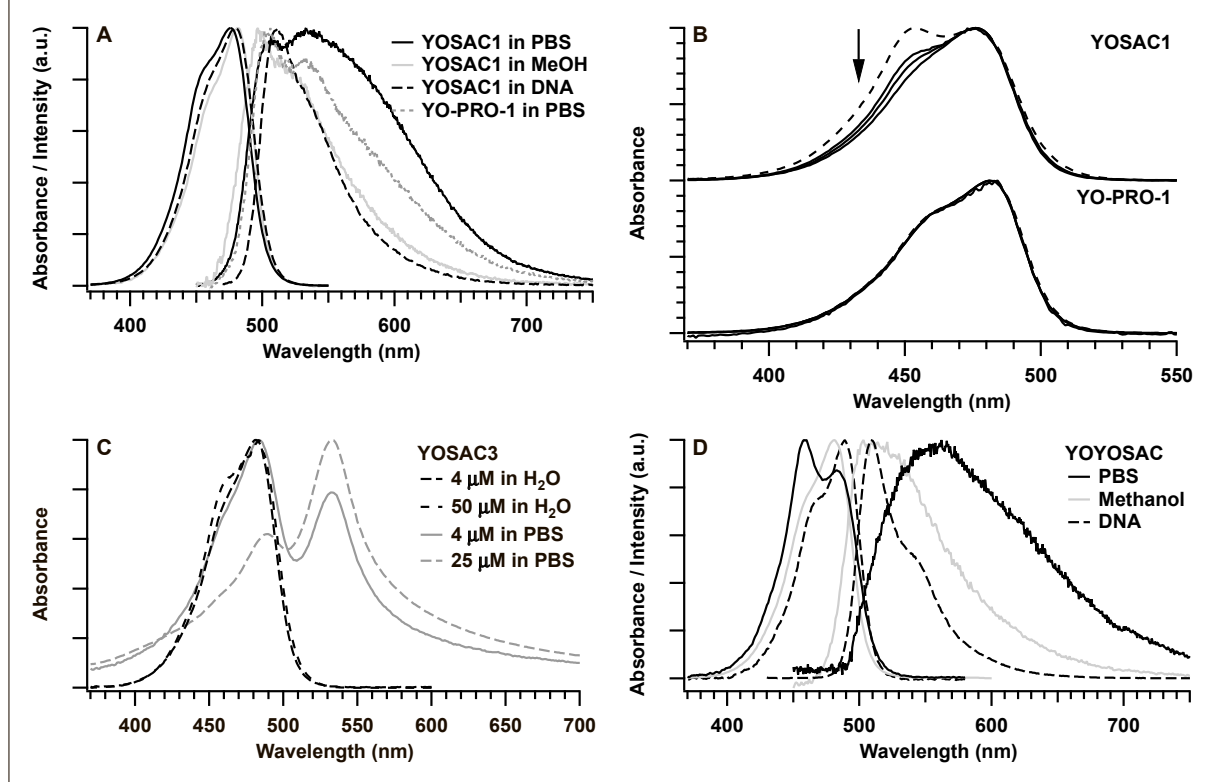

Fig. 3. (A) Intensity-normalized absorption and fluorescence spectra of YOSAC1 in PBS, methanol, and DNA and fluorescence spectrum of YO-PRO-1 in PBS. (B) Intensity-normalized absorption spectra of a $50 \mu \mathrm{M}$ solution of YOSAC1 (top) and YO-PRO-1 (bottom) in water diluted down (arrow) to $3.1 \mu \mathrm{M}$ for YOSAC1 and $6.2 \mu \mathrm{M}$ for YO-PRO-1 (solid lines). The dashed lines correspond to concentrations of $200 \mu \mathrm{M}$ in PBS. (C) Intensity-normalized absorption spectra of YOSAC3 at different concentrations in pure water and in PBS. (D) Intensity-normalized absorption and fluorescence spectra of YOYOSAC in PBS, methanol, and DNA.

On the other hand, the structurally very similar dye YO-PRO-1 displays much lower propensity to aggregate at similar concentrations as the shoulder around 460 $\mathrm{nm}$ in the absorption spectrum in water stays constant (Fig. 3B, bottom). However, also for this dye the fluorescence spectrum in aqueous solution is broader than in organic solvents (Fig. 3A) and it was shown that it is concentration and excitation wavelength dependent. [15] Nonetheless it is not as broad as in the case of YOSAC1 and the fluorescence quantum yield of free YO-PRO-1 is half that of YOSAC1 (Table $1)^{[15]}$ Since both the aggregated and nonaggregated dye populations contribute to the fluorescence spectrum and thus to the fluorescence quantum yield, it can be concluded that the aggregates contribute more to the fluorescence of the free dyes than the non-aggregated dyes. ${ }^{[15]}$ The relevant structural difference here between YOSAC1 and YO-PRO-1 is that, whereas YOSAC1 carries a single positive charge, YO-PRO-1 possesses two positive charges, which seem to have a beneficial effect on the solubility of the dye monomer in aqueous solution.

Interestingly, contrary to YOSAC1 and YO-PRO-1, the structurally very similar dye YOSAC3, which also has a single positive charge, is able to form both $\mathrm{H}$ - and J-aggregates in an aqueous environment depending on the ionic strength of the solution (Fig. 3C). ${ }^{[14 b]}$ In pure water, it displays an absorption spectrum very similar to that of YOSAC1, with an
$\mathrm{H}$-aggregate band growing with increasing dye concentration and a similarly low fluorescence quantum yield. In aqueous buffer solution however, fluorescent J-aggregates form in a concentrationdependent way. YOSAC3 is the only dye of its kind to have a chlorine substituent and the presence of chlorine atoms on cyanines has been shown to facilitate the formation of J-aggregates. ${ }^{[16]}$

In the examples presented so far, aggregation relied on the serendipitous encounter of two dye molecules by diffusion and therefore depends on the dye concentration. But two monomers can also be brought and kept in proximity of each other by covalent coupling through a linker, as it is the case with the homodimeric DNA intercalators of the YOYO family. For the homodimer YOYOSAC, the absorption spectra in organic solvents like methanol display a maximum around $490 \mathrm{~nm}$ and a vibronic shoulder around $465 \mathrm{~nm}$ (Fig. $3 \mathrm{D}),{ }^{[14 a]}$ very much like the monomeric the bound and the free form. cyanine dyes described above. In aqueous solution, however, the absorption is characterized by two maxima around 460 and $485 \mathrm{~nm}$ whose relative intensity does not depend on the dye concentration. The blue-shifted peak at $460 \mathrm{~nm}$ can therefore be attributed to intramolecular H-dimers formed between the two chromophoric moieties of the molecule. Aggregation is strongly favoured through the preorganization induced by the covalent linker which removes the diffusion step which controls aggregation of monomers in dilute solution. The fluorescence spectrum of YOYOSAC in water is also broad and red-shifted compared to methanol (Fig. $3 \mathrm{D})$ and the fluorescence quantum yield of the free dye, consistently with the fact that intramolecular aggregation is more efficient than intermolecular aggregation, is nearly three times higher than that of YOSAC1.

In the presence of DNA, all of the described dyes strongly bind to it, mostly through intercalation. ${ }^{[13]}$ As a consequence of binding, $\mathrm{H}$-aggregates are disrupted, the fluorescence spectra become mirror images of the absorption spectra and the fluorescence quantum yield strongly increases because of the constrictive DNA environment which prevents torsional non-radiative deactivation of the excited state. $[14,15]$ It should be noted that in the case of YOYOSAC and other homodimeric DNA intercalators of the same type, disruption of the excitonic interaction in DNA is more due to their quasi-orthogonal relative orientation (NMR measurements of TOTO-1 bound to DNA indicate a dihedral angle of $83^{\circ}$ between the chromophoric units) ${ }^{[17]}$ than to the distance between the chromophores.[14a] However, the fluorescence contrast, that is the difference in fluorescence quantum yield between the free and the DNA-bound forms, is worse for YOYOSAC than for YOSAC1 than for YO-PRO-1 (Table 1). This is due to the fact that the higher the fluorescence quantum yield of the free form, the worse the fluorescence contrast, the magnitude of which is therefore anti-correlated with the propensity of the dyes to form $\mathrm{H}$-dimers. This indicates that aggregation

Table 1. Fluorescence quantum yield $\left(\Phi_{\mathrm{fl}}\right)$ of given DNA intercalators in aqueous buffer solution and when bound to DNA. ${ }^{15]}$ The last column indicates the fluorescence enhancement between

\begin{tabular}{l|l|ll} 
Dye & $\Phi_{\mathrm{n}}($ free $) \times \mathbf{1 0}^{\mathbf{4}}$ & $\Phi_{\mathrm{n}}($ DNA $)$ & Contrast \\
\hline YOSAC1 & 5.3 & 0.35 & 620 \\
YO-PRO-1 & 2.4 & 0.42 & 1660 \\
\hline YOSAC3 & $9.7\left(\right.$ in $\left.\mathrm{H}_{2} \mathrm{O}\right)$ & 0.61 & 590 \\
YOYOSAC & 14 & 0.41 & 290
\end{tabular}


is not as efficient as isomerization as a fluorescence contrast mechanism, at least for these cyanine dyes, and therefore that it should be avoided in order to optimize the fluorescence contrast of this dye family. ${ }^{[15]}$ In the case of the monomeric dyes of the YO type, this can be achieved by designing derivatives with a maximized number of positive charges.

Aggregates can also form in aqueous solution with dye molecules which do not possess a planar core. This is for example the case of chiral [4]-helicene cation derivatives which coexist in aqueous solution as monomers and dimeric aggregates. ${ }^{[18]}$ With this family of dyes, both the monomers and the dimers are able to bind to DNA, the former probably by intercalation and the latter by groove binding.[19] The contrast achieved (3-4) is much lower than with the cyanine DNA intercalators, but a stereoselectivity was observed with the binding constants for the $M$-enantiomers of the helicene derivatives being systematically higher than for the $P$-derivatives. In the case of these dyes, however, the photophysics of the monomers and of the dimers cannot be as clearly disentangled as with the cyanine dyes, which renders a quantitative application of the helicene derivatives more problematic.

Finally, aggregation can also occur when two or multiple copies of the same fluorophore used to label a biomolecule of interest find each other at such close proximity on the biomolecule that an excitonic interaction between them becomes possible. This situation may for example occur upon labelling of a protein with an amine-reactive dye on multiple lysine residues, as illustrated in Fig. 4. The amine-reactive version of the dye ATTO680 was used to label lysine side chains of the apolipoprotein A1 in recombinant high-density lipoparticles ${ }^{[20]}$ and two samples were prepared. The ATTO680 concentration used for the labelling reaction was six times higher with the second sample than with the first. The absorption spectra of these two samples after removal of the unreacted dye clearly show that, in the case of the second sample, the labelling density on the protein is so high that $\mathrm{H}$-dimers form, whereas the absorption spectrum of the first sample is very similar to that of free ATTO680 in water. When the purpose of such fluorescence labelling is the mere detection of the probe, then aggregation like in this example is unlikely to cause any problems. However, if the fluorescence intensity, the fluorescence quantum yield or the absorbance are to be used quantitatively, as it is the case in the determination of the degree of labelling or in some single-molecule counting applications, then aggregation will flaw the evaluation. The best way to assess whether dye aggregation is taking place or not is to compare the absorption spectrum of the labelled sample with that of the free dye. Any shoulder which is more pronounced than in the dilute free dye spectrum will be indicative of aggregation. For quantitative applications, a new sample should be prepared with a smaller quantity of reactive dye.

It should finally be noted that, instead of aggregating as a consequence of a too low concentration or a lack

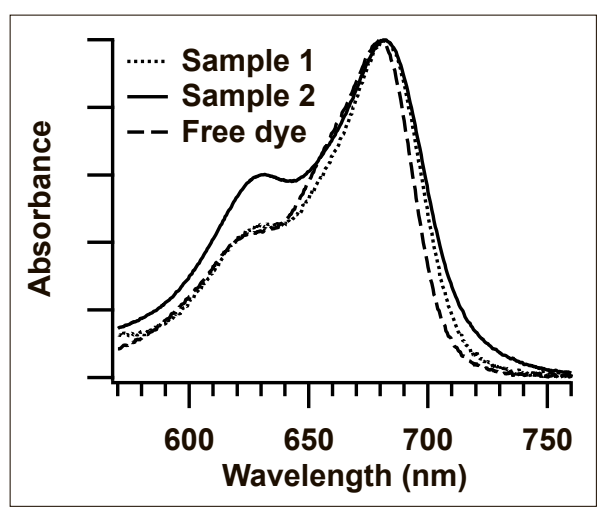

Fig. 4. Intensity-normalized absorption spectra of ATTO680 coupled to the lysine residues of apolipoprotein $\mathrm{A} 1$ at various labelling concentrations (samples 1 and 2) and in free aqueous solution (dashed line).

of repulsive electrostatic interactions between dye molecules, water soluble organic fluorophores may seek interaction with other non-chromophoric organic partners in their surroundings driven by the same hydrophobic interaction as in the aggregation process. It has for example been shown that the biotin-attached dye Lucifer Yellow bound to the protein avidin interacts directly with the protein surface instead of sticking out in the water despite a long, flexible linker, ${ }^{[21]}$ and that many water-soluble fluorophores tend to interact with lipid bilayers instead of staying in water when they have the possibility. ${ }^{[22]}$

\section{Water as a Quencher of Fluorescence}

One of the most overlooked and yet intriguing effects of water as a solvent for fluorescence spectroscopy is that it can reduce the fluorescence quantum yield of a dye, that is water can act as a quencher of the fluorescence. This is again well exemplified with the oxazine dye ATTO680 (Fig. 5B). Together with the dyes ATTO660 and ATTO700, this dye belongs to a new generation of popular oxazines compatible with bioimaging[23] thanks to their increased water solubility and reactive functionalities which enable directed covalent coupling to targets of interest. These dyes display little solvatochromism. ATTO680 has its absorption maximum between 670 and $690 \mathrm{~nm}$ depending on the solvent and its emission maximum between 690 and $710 \mathrm{~nm}$. In polar aprotic organic solvents such as acetonitrile, DMF, acetone, or DMSO, its fluorescence quantum yield ranges between 0.46 and 0.53 (Fig. 5A). ${ }^{[24]}$ In alcohols however, the fluorescence quantum yield decreases, with a value of 0.39 in ethanol and 2-propanol and a value of 0.34 in methanol. In water $\left(\mathrm{H}_{2} \mathrm{O}\right)$, its magnitude drops even to 0.19 . Interestingly, in heavy water $\left(\mathrm{D}_{2} \mathrm{O}\right)$, the fluorescence quantum yield is $0.43,2.3$ times higher than in $\mathrm{H}_{2} \mathrm{O}$ and very close to the values observed in non-protic, polar organic solvents. Qualitatively analogous observations were made with the structurally related dyes ATTO655 and ATTO700. [24] Furthermore, with all three dyes and in all solvents, the modulation of the excited-state lifetime perfectly followed the trend observed with the fluorescence quantum yield, indicating that the radiative rate stays constant. [24] An enhancement of the fluorescence in $\mathrm{D}_{2} \mathrm{O}$ compared to $\mathrm{H}_{2} \mathrm{O}$ was also reported for a series of cyanine dyes. ${ }^{[25]}$ Whereas the fluorescence quantum yield and lifetime increased by less than $10 \%$ with the dye $\mathrm{Cy} 3$, they were about 1.3-fold higher for Cy5 and Alexa Fluor 647 and 2.6-fold higher for Cy7 in $\mathrm{D}_{2} \mathrm{O}$ than in $\mathrm{H}_{2} \mathrm{O}$ (Fig. 6). Overall these observations led to the conclusion that a non-radiative deactivation pathway is selectively operative in hydrogenbonding solvents, in particular $\mathrm{H}_{2} \mathrm{O}$, and exclusively responsible for the reduction of the fluorescence quantum yield and of the excited-state lifetime (Fig. 5C).[24,25]

Quenching of fluorescence by $\mathrm{H}_{2} \mathrm{O}$ has been reported for many dyes from different classes including oxazines, cyanines, rhodamines, carborhodamines, aminonaphthalimides, or fluorescent proteins to name a few, ${ }^{[6,24-26]}$ and a deuterium isotope effect on the fluorescence quantum yield of dyes was first reported as early as 1966. [27] This hydrogen-bond-assisted nonradiative deactivation of the excited state can be thought of as an efficient internal conversion process in which the vibrational modes of the hydrogen bonds between water and the dye act as accepting modes of the non-radiative transition from the $S_{1}$ to the ground state of the fluorophore. ${ }^{[25,28]}$ The efficiency of this non-radiative deactivation is greatly reduced in $\mathrm{D}_{2} \mathrm{O}$ because the vibrational modes exhibit lower energies, leading to lower Frank-Condon factors. The observation for cyanine dyes that the isotope effect is enhanced as the dye absorption shifts to the red (Fig. 6) was further attributed to a resonance between the 


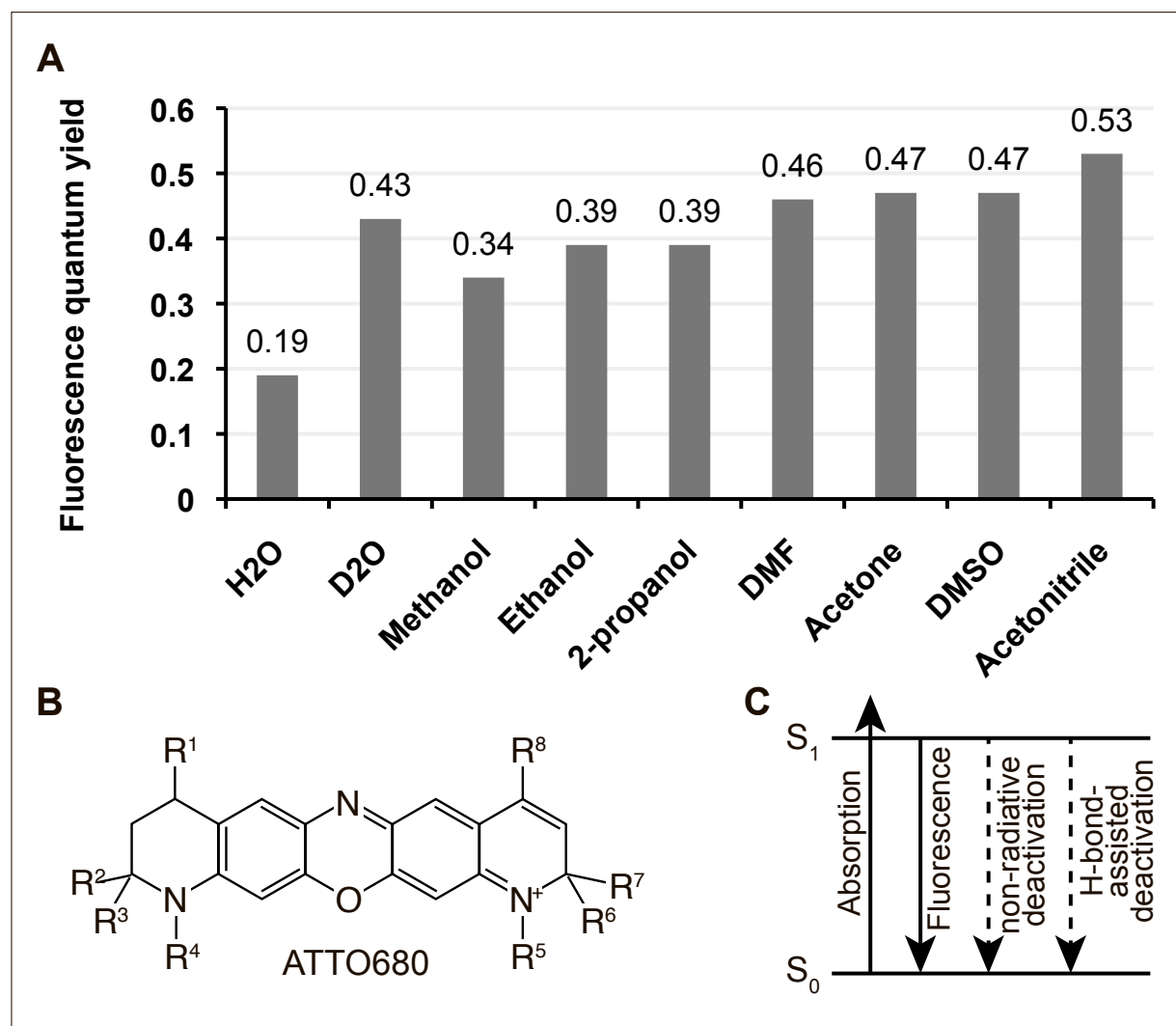

Fig. 5. (A) Fluorescence quantum yield of ATTO680 in various solvents. (B) Molecular structure of ATTO680. (C) Proposed Jablonski diagram. In $\mathrm{H}_{2} \mathrm{O}$, hydrogen bonding causes strong nonradiative deactivation in addition to other non-radiative and radiative pathways.

emission frequency of the cyanines and vibrational overtones of the $\mathrm{H}_{2} \mathrm{O}$ molecule ${ }^{[25]}$ responsible for the weak absorption bands in the visible spectrum, ${ }^{[29]}$ which is expected to favour energy dissipation through the solvent via solvent molecules closely interacting with the dye. These overtones are at much lower energy with $\mathrm{D}_{2} \mathrm{O}$, which consequently does not have any absorption band in the visible part of the electromagnetic spectrum. It should further be stressed that, although $\mathrm{H}_{2} \mathrm{O}$ is a quencher of many dyes, it is a relatively weak quencher: the dynamic quenching constant extracted from an experiment in which the $\mathrm{H}_{2} \mathrm{O}$ concentration was varied in a solution of ATTO655 in $\mathrm{D}_{2} \mathrm{O}$ is on the order of $3 \times 10^{6}$ $\mathrm{M}^{-1} \mathrm{~s}^{-1}$, which is three orders of magnitude below the diffusion-limited quenching rate constant in water. It is nonetheless effective at quenching fluorophores because of its high concentration.

Hydrogen-bond-assisted non-radiative deactivation therefore seems generally operative with oxazines, cyanines and other dye classes in aqueous environments but the quenching of fluorescence can be strongly reduced by replacing $\mathrm{H}_{2} \mathrm{O}$ by $\mathrm{D}_{2} \mathrm{O}$. The usefulness of this phenomenon was demonstrated in several applications of fluorescence imaging ${ }^{[24,25,30]}$ in which fluorophore brightness is an essential parameter. Indeed, the brighter a fluorophore, the better the signal-to-back-
A

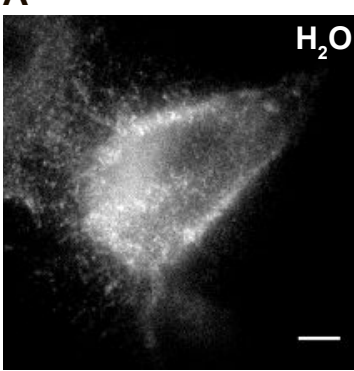

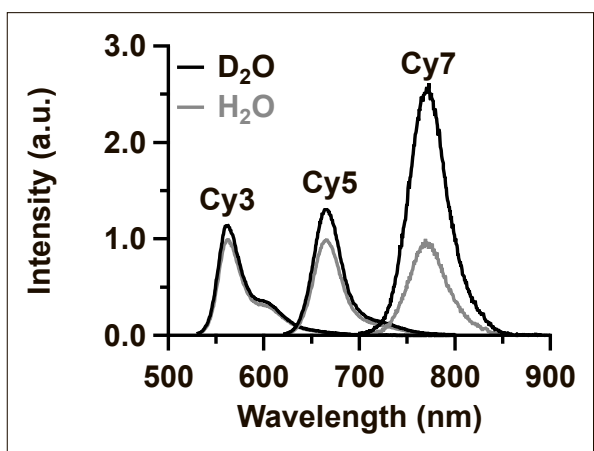

Fig. 6. Fluorescence emission spectra of the cyanine dyes $\mathrm{Cy} 3, \mathrm{Cy} 5$, and $\mathrm{Cy} 7$ in $\mathrm{H}_{2} \mathrm{O}$ and in $\mathrm{D}_{2} \mathrm{O}$. For better comparison, the fluorescence intensity in $\mathrm{H}_{2} \mathrm{O}$ was set to 1 for all dyes.

better the resolution is in the final image. Fig. 7 shows fixed mammalian cells stably expressing the $\mathrm{C}-\mathrm{C}$ chemokine receptor 5 (CCR5), a membrane protein belonging to the $\mathrm{G}$ protein-coupled receptor family. CCR5 was stained with a primary antibody labelled with the oxazine dye ATTO655 and imaged under the same conditions by wide-field fluorescence microscopy in $\mathrm{H}_{2} \mathrm{O}$ and in $\mathrm{D}_{2} \mathrm{O}$ (Fig. 7A and 7B). [24] The image recorded in $\mathrm{D}_{2} \mathrm{O}$ was significantly brighter, as expected from the 2.1-fold increase in fluorescence quantum yield measured with ATTO655 in $\mathrm{H}_{2} \mathrm{O}$ and $\mathrm{D}_{2} \mathrm{O}$. ${ }^{[24]}$

Furthermore, it was demonstrated that the enhancement in fluorescence quantum yield in $\mathrm{D}_{2} \mathrm{O}$ also corresponded to an increased number of photons from individual fluorophores: the number of photons recorded from immobilized single ATTO655 molecules on a glass surface was approximately twice as high in $\mathrm{D}_{2} \mathrm{O}$ as in $\mathrm{H}_{2} \mathrm{O}$, resulting in an improved precision with which single molecules could be localized.[24] An improved photon yield in $\mathrm{D}_{2} \mathrm{O}$ was also observed with Alexa Fluor 647 molecules $^{[25]}$ and with different photoactivatable fluorescence proteins ${ }^{[26 c]}$ when they were imaged at the singlemolecule level.

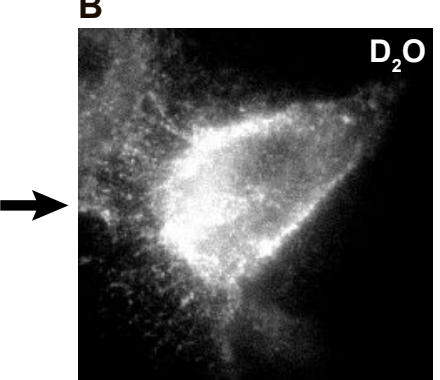

C

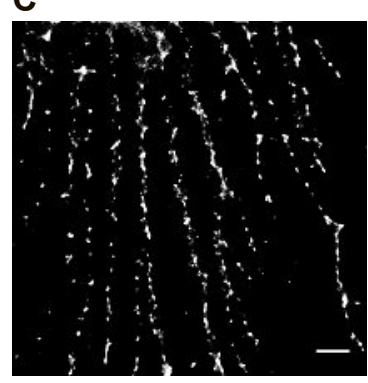

Fig. 7. (A, B) Fixed mammalian cells expressing CCR5 stained with an ATTO655-labelled antibody against CCR5 and imaged under identical conditions first in $\mathrm{H}_{2} \mathrm{O}(A)$ and then in $\mathrm{D}_{2} \mathrm{O}(\mathrm{B})$. Images are shown at constant contrast. Scale bar: $5 \mu \mathrm{m}$. (C) Super-resolution image of filopodia of mammalian cells. The filopodia were immunostained using the same ATTO655-labeled antibody against CCR5 and imaged by dSTORM in $\mathrm{D}_{2} \mathrm{O}$. Scale bar: $1 \mu \mathrm{m}$. 
Finally, it was shown in several instances that super-resolution imaging in $\mathrm{D}_{2} \mathrm{O}$ with ATTO655 could be used to resolve diffraction-limited subcellular structures such as filopodia of mammalian cells $^{[24]}$ (Fig. 7C) and intracellular clusters of arrestin proteins.[30] Features with peak-to-peak distances as small as 128 $\mathrm{nm}$ could be clearly resolved and protein clusters $60 \mathrm{~nm}$ in size could be imaged with a localization precision of about 20 $\mathrm{nm}$. Experiments were performed exactly in the same way as in $\mathrm{H}_{2} \mathrm{O}$ except that the imaging buffer which was added just before the experiment had been prepared in $\mathrm{D}_{2} \mathrm{O}$ instead of $\mathrm{H}_{2} \mathrm{O}$. Microtubules of HeLa cells stained with an Alexa Fluor 647 labelled antibody could also be clearly resolved in dSTORM experiments in $\mathrm{D}_{2} \mathrm{O} .{ }^{[25]}$

The observations described in this section suggest that it would be beneficial to generally perform fluorescence imaging, in particular super-resolution microscopy, in $\mathrm{D}_{2} \mathrm{O}$ instead of $\mathrm{H}_{2} \mathrm{O}$. Indeed, for many dyes like oxazines and cyanines more photons come out per time bin in $\mathrm{D}_{2} \mathrm{O}$, allowing in principle for shorter imaging times. In addition, $\mathrm{D}_{2} \mathrm{O}$ has otherwise no detrimental effect on super-resolution imaging with cyanines and oxazines and is cost-effective, non-toxic and live-cell compatible.

\section{Conclusion}

Although water is the solvent of life, it is not a totally 'innocent' solvent when fluorescence spectroscopy and imaging experiments are to be considered. I have discussed and illustrated through several examples the phenomena of organic fluorophore aggregation in water and of fluorescence quenching by water which can lead to difficulties in the interpretation and the quantitative assessment of fluorescence data. In addition, I have pointed out some ways to overcome or to solve them. Dye aggregation is not so much of a problem when the purpose of the experiment is the detection of a sample by absorption or fluorescence. However, since aggregation has a strong effect on the absorption and on the fluorescence spectra of a dye, it should be avoided or taken into account when such an experiment is meant to provide quantitative information. Aggregation can be minimized by choosing or designing chromophores which will naturally repel each other through electrostatic interactions. On the other hand, fluorescence quenching by water happens with most available chromophores and will often reduce the fluorescence quantum yield of a dye in water compared to polar organic solvents. In biomolecular fluorescence experiments, increasing the brightness of a probe by working in organic solvents is most of the time not an option, but the fluorescence of many dyes increases in heavy water almost to the level observed in organic solvents. In many instances, it is possible to replace $\mathrm{H}_{2} \mathrm{O}$ by $\mathrm{D}_{2} \mathrm{O}$ because $\mathrm{D}_{2} \mathrm{O}$ is only required at the time of the measurement. Measurements in $\mathrm{D}_{2} \mathrm{O}$ represent therefore a very simple and cost-effective way to improve the signal in fluorescence experiments which are then nonetheless still performed in an aqueous and almost native environment.

Received: January 17, 2017

[1] a) X. Michalet, A. N. Kapanidis, T. Laurence, F. Pinaud, S. Doose, M. Pflughoefft, S. Weiss, Annu. Rev. Biophys. Biomol. Struct. 2003, 32, 161 ; b) H. Tian, A. Fürstenberg, T. Huber, Chem. Rev. 2017, 117, 186; c) C. Joo, H. Balci, Y. Ishitsuka, C. Buranachai, T. Ha, Аnпи. Rev. Biochem. 2008, 77, 51.

[2] a) W. E. Moerner, Angew. Chem. Int. Ed. 2015, 54, 8067; b) X. Michalet, S. Weiss, M. Jager, Chem. Rev. 2006, 106, 1785; c) G. Patterson, M. Davidson, S. Manley, J. Lippincott-Schwartz, Annu. Rev. Phys. Chem. 2010, 61, 345.

[3] L. D. Lavis, R. T. Raines, ACS Chem. Biol. 2008, 3,142 .

[4] R. Y. Tsien, Angew. Chem. Int. Ed. 2009, 48, 5612.

[5] a) M. S. Goncalves, Chem. Rev. 2009, 109, 190; b) Y.-X. Chen, G. Triola, H. Waldmann, Acc. Chem. Res. 2011, 44, 762; c) T. Huber, T. P. Sakmar, Chem. Biol. 2014, 21, 1224.

[6] a) A. Fürstenberg, E. Vauthey, Chimia 2007, 61, 617; b) A. Fürstenberg, E. Vauthey, Photochem . Photobiol. Sci. 2005, 4, 260; c) S. Bhosale, A. L. Sisson, P. Talukdar, A. Fürstenberg, N. Banerji, E. Vauthey, G. Bollot, J. Mareda, C. Roeger, F. Würthner, N. Sakai, S. Matile, Science 2006, 313, 84; d) S. Bockenhauer, A. Fürstenberg, X. J. Yao, B. K. Kobilka, W. E. Moerner, J. Phys. Chem. B 2011, 115, 13328.

[7] L. D. Lavis, R. T. Raines, ACS Chem. Biol. 2014, 9,855 .

[8] J. Knof, F.-J. Theiss, J. Weber, Z. Naturfosch. 1978, 33a, 98 .

[9] a) M. Kasha, H. R. Rawls, M. A. El-Bayoumi, Pure. Appl. Chem. 1965, 11, 371; b) A. S. Davydov, 'Theory of Molecular Excitons', Plenum Press, New York, 1971.

[10] a) A. Mishra, R. K. Behera, P. K. Behera, B. K. Mishra, G. B. Behera, Chem. Rev. 2000, 100, 1973; b) F. Lopez Arbeloando, I. Llona Gonzalez, P. Ruiz Ojeda, I. Lopez Arbeloa, $J$. Chem Soc. Farad. Trans. 1982, 78, 989.

[11] P. Selvin, Science 1992, 257, 885.

[12] a) H. S. Rye, M. A. Quesada, K. Peck, R. A. Mathies, A. N. Glazer, Nucleic Acids Res. 1991, 19,327 ; b) H. S. Rye, S. Yue, D. E. Wemmer, M. A. Quesada, R. P. Haugland, R. A. Mathies, A. N. Glazer, Nucleic Acids Res. 1992, 20 , 2803.

[13] C. Carlsson, A. Larsson, M. Jonsson, B. Albinsson, B. Norden, J. Phys. Chem. 1994, 98 , 10313.

[14] a) A. Fürstenberg, M. D. Julliard, T. G. Deligeorgiev, N. I. Gadjev, A. A. Vasilev, E. Vauthey, J. Am. Chem. Soc. 2006, 128, 7661; b) A. Fürstenberg, E. Vauthey, J. Phys. Chem. $B$ 2007, 111, 12610 .

[15] A. Fürstenberg, T. G. Deligeorgiev, N. I. Gadjev, A. A. Vasilev, E. Vauthey, Chem. Eur. J. 2007, 13, 8600 .
[16] M. Iwasaki, K. Higashinaka, T. Tanaka, J. Soc. Photogr. Sci. Technol. Jpn 1995, 58, 361.

[17] H. P. Spielmann, D. E. Wemmer, J. P. Jacobsen, Biochemistry 1995, 34, 8542

[18] O. Kel, P. Sherin, N. Mehanna, B. Laleu, J. Lacour, E. Vauthey, Photochem. Photobiol. Sci. 2012, 11, 623 .

[19] O. Kel, A. Fürstenberg, N. Mehanna, C. Nicolas, B. Laleu, M. Hammarson, B. Albinsson, J. Lacour, E. Vauthey, Chem. Eur. J. 2013, 19, 7173.

[20] M. R. Whorton, M. P. Bokoch, S. G. F. Rasmussen, B. Huang, R. N. Zare, B. Kobilka, R. K. Sunahara, Proc. Natl. Acad. Sci. USA 2007, 104, 7682

[21] A. Fürstenberg, O. Kel, J. Gradinaru, T. R Ward, D. Emery, G. Bollot, J. Mareda, E. Vauthey, ChemPhysChem 2009, 10, 1517.

[22] L. D. Hughes, R. J. Rawle, S. G. Boxer, PLoS One 2014, 9.

[23] M. Heilemann, S. van de Linde, A. Mukherjee, M. Sauer, Angew. Chem. Int. Ed. 2009, 48, 6903.

[24] S. F. Lee, Q. Vérolet, A. Fürstenberg, Angew. Chem. Int. Ed. 2013, 52, 8948.

[25] K. Klehs, C. Spahn, U. Endesfelder, S. F. Lee, A. Fürstenberg, M. Heilemann, ChemPhysChem 2014, 15, 637.

[26] a) R. Sens, K. H. Drexhage, J. Lumin. 1981, 24 25, 709; b) H. Inoue, M. Hida, N. Nakashima, K. Yoshihara, J. Phys. Chem. 1982, 86, 3184; c) W. Q. Ong, Y. R. Citron, J. Schnitzbauer, D. Kamiyama, B. Huang, Chem. Commun. 2015, $51,13451$.

[27] L. Stryer, J. Am. Chem. Soc. 1966, 88, 5708.

[28] a) A. Grofcsik, M. Kubinyi, W. J. Jones, Chem. Phys. Lett. 1996, 250, 261; b) P. Fita, M. Fedoseeva, E. Vauthey, J. Phys. Chem. A 2011, $115,2465$.

[29] C. L. Braun, S. N. Smirnov, J. Chem. Educ. 1993, 70,612 .

[30] a) Z. Truan, L. Tarancón Díez, C. Bönsch, S. Malkusch, U. Endesfelder, M. Munteanu, O. Hartley, M. Heilemann, A. Fürstenberg, $J$. Struct. Biol. 2013, 184, 329; b) L. Tarancon Diez, C. Bönsch, S. Malkusch, Z. Truan, M. Munteanu, M. Heilemann, O. Hartley, U. Endesfelder, A. Fürstenberg, Histochem. Cell. Biol. 2014, 142, 69.

[31] A. Fürstenberg, M. Heilemann, Phys. Chem Chem. Phys. 2013, 15, 14919.

[32] E. Betzig, G. H. Patterson, R. Sougrat, O. W. Lindwasser, S. Olenych, J. S. Bonifacino, M. W. Davidson, J. Lippincott-Schwartz, H. F. Hess, Science 2006, 313, 1642.

[33] M. Heilemann, S. van de Linde, M. Schuttpelz, R. Kasper, B. Seefeldt, A. Mukherjee, P. Tinnefeld, M. Sauer, Angew. Chem. Int. Ed. 2008, 47, 6172 .

[34] R. E. Thompson, D. R. Larson, W. W. Webb, Biophys. J. 2002, 82, 2775. 